\title{
Barriers to Secondary Stroke Prevention and Care in Urban and Rural Uganda: Lived Experiences of Stroke Survivors, Carers and Key Stakeholders
}

\author{
Jane Nakibuuka, ${ }^{1}$, Haddy Nalubwama ${ }^{2}$, Elizabeth Nambalirwa ${ }^{3}$, Olivia Namusoke ${ }^{4}$, \\ Jayne Byakika-Tusiime ${ }^{5}$, Martha Sajatovic ${ }^{6}$, Elly Katabira ${ }^{7}$ \\ ${ }^{1}$ Department of Medicine, Mulago National Referral Hospital, Kampala, Uganda \\ ${ }^{2}$ Department of Obstetrics and Gynaecology, School of Medicine, Makerere University College of Health Sciences, Kampala, Uganda \\ ${ }^{3}$ Division of Social Sciences, Makerere Institute of Social Research, Kampala, Uganda \\ ${ }^{4}$ Infectious Diseases Institute, Makerere University College of Health Sciences, Kampala, Uganda \\ ${ }^{5}$ Emergency Preparedness Program, WHO Regional Office ESA Hub, Nairobi, Kenya \\ ${ }^{6}$ Neurological and Behavioral Outcomes Center, University Hospitals Case Medical Center, Case Western Reserve University, School of \\ Medicine, Cleveland Ohio, USA \\ ${ }^{7}$ Department of Medicine, School of Medicine, Makerere University College of Health Sciences, Kampala, Uganda
}

\section{Email address:}

nakibuukajm@yahoo.com (J. Nakibuuka),hnalubwama@yahoo.com (H. Nalubwama), elizabethbwete@gmail.com (E. Nambalirwa), olivenam2014@gmail.com (O. Namusoke),jayne.tusiime@gmail.com (J. Byakika-Tusiime),

Martha.sajatovic@uhhospitals.org (M. Sajatovic),Katabira@infocom.co.ug (E. Katabira)

${ }^{*}$ Corresponding author

\section{To cite this article:}

Jane Nakibuuka, Haddy Nalubwama, Elizabeth Nambalirwa, Olivia Namusoke, Jayne Byakika-Tusiime, Martha Sajatovic, Elly Katabira. Barriers to Secondary Stroke Prevention and Care in Urban and Rural Uganda: Lived Experiences of Stroke Survivors, Carers and Key Stakeholders. Clinical Medicine Research. Vol. 10, No. 3, 2021, pp. 59-66. doi: 10.11648/j.cmr.20211003.11

Received: April 6, 2021; Accepted: April 19, 2021; Published: May 8, 2021

\begin{abstract}
Although Stroke is largely preventable, it remains a major public health concern in Uganda which calls for inquiry into the factors impeding its prevention and management in both urban and rural settings. This study explored barriers to secondary stroke prevention and care based on lived experiences of stroke survivors, their care takers and key stakeholders, to provide a broader picture of key areas that require modification to achieve successful stroke prevention and care programs. A total of 31 participants (stroke survivors, care takers, key stakeholders) stratified by age and sex were engaged in in-depth and key informant interviews in urban and rural populations of Wakiso district in central Uganda. Interviews were audio recorded and transcribed verbatim. Transcripts were analyzed thematically using inductive and deductive approaches. Three major domains emerged from the interview data: 1) Patient level barriers (inadequate stroke knowledge, financial impediments, posttraumatic stress disorder, pill burden, adamancy when feeling better), 2) Community level barriers (limited social support, stigmatization), 3) Health system level barriers (scarcity of human resources and drugs, poor patient-health worker relationship, poor access to healthcare, lack of stroke rehabilitation centres and equipment). Rural participants especially insisted on improving stroke awareness and access to healthcare to improve prevention and care programs. In conclusion, stroke prevention and care program interventions must address barriers at individual and community levels where increased stroke awareness should be emphasized and at health system level where access to healthcare facilities, human resource and equipment are improved to meet stroke specific needs.
\end{abstract}

Keywords: Secondary Prevention, Stroke Survivors, Care Takers, Key Informants, Stroke, Uganda 


\section{Introduction}

Stroke is emerging as a leading cause of preventable death and disability worldwide with an estimated annual prevalence of 15 million sufferers, among whom nearly 5.4 million die and another 5 million are left permanently disabled [1-9]. This puts a high burden on families/communities and economies especially in low-middle income countries (LMICS) that currently account for $85 \%$ of the global deaths from stroke and the disability adjusted life years (DALYs) lost, nearly seven times higher than in high income countries (HIC) $[1,2,5,8,9]$. In Uganda, the stroke burden is soaring with 2018 statistics ranking stroke as the $9^{\text {th }}$ top most cause of death [10] compared to the $14^{\text {th }}$ rank in 2013 and $19^{\text {th }}$ rank in 1990 according to the 2014 World Health Organisation (WHO) estimates [2]. The increase in stroke burden could be largely attributed to an increase in the population, improved life expectancy, adoption of unhealthy lifestyles like smoking, physical inactivity, unhealthy diets, high blood cholesterol and an increase in non-communicable diseases such as high blood pressure [1, 9, 11]. High blood pressure which is the most potent risk factor for stroke is prevalent in $25.5 \%$ urban and 24.4\% rural communities in Uganda [12-14]. Fortunately, stroke risk factors are largely modifiable which makes stroke occurrence and recurrence largely preventable. However, what remains unexplained is why the prevalence of stroke and its major risk factors such as high blood pressure remains high. This warrants exploration of the impediments to stroke prevention and care. Multiple studies on this subject have been conducted mainly in urban/semi urban populations [15-21]. These studies reported knowledge barriers, financial constraints, post-traumatic stress disorders (PTSD), community support barriers and health system barriers such as health worker attitude, long waiting hours and poor access to health facilities as impediments to stroke prevention and care [15-21]. In Uganda, there is paucity of data in this area. Literature search revealed only two studies that were conducted among urban/semi urban communities [18, 19]. In reference [18], stroke survivors, caregivers and those at high risk for stroke were interviewed while in reference [19], care givers were interviewed. Compared to studies in HICs, these studies reported additional individual barriers such as resistance to seeking care and medication nonadherence as well as family barriers such as caretaker burden. These barriers to stroke prevention could further differ from rural populations, making a study combining both urban and rural populations necessary.

This study sought to explore the barriers to secondary stroke prevention and care in the perspectives of stroke survivors, their caretakers and key stakeholders from urban and rural populations. Findings are expected to provide a broader scope of knowledge to inform development of stroke prevention and care programs tailored to the needs of the populations in both social economic settings.

\section{Methods}

\subsection{Study Design}

This was a cross sectional qualitative study.

\subsection{Study Setting}

The study was conducted in urban Nansana sub-county and rural Busukuma sub-county, Wakiso district in central Uganda. Wakiso district which is the second most populous district in Uganda, surrounds the capital city Kampala with peri-urban neighbourhoods bordering the city to typically rural areas.

\subsection{Study Population and Sampling Strategy}

Thirty one study participants were recruited over a period of two months from February to March 2013 from a sample of 5420 urban and rural participants enrolled in a cross sectional survey to determine risk factors for stroke in urban and rural populations in Wakiso district in central Uganda between August 2012 and August 2013 [14]. The study participants consisting of 8 stroke survivors, 8 caretakers to stroke survivors and 15 key stakeholders stratified by age ( $\leq 45$ years, $>45$ years) and gender were purposively recruited from within their communities with the help of village health team (VHT) members who were deemed knowledgeable about the residents of their communities. Inclusion criteria was age 18 years or older, with intact cognition and able to provide written informed consent. The communities in the urban sub-county of Nansana town council that were sampled included Nansana, Kazo, Kawaala, Nabweru, Lugooba and Nakuule, while in the rural sub-county of Busukuma, they included Busukuma, Kiwenda, Buwagga, Magigye, Namulonge, Buwagga, and Kyambogo-Nabutiti.

Four stroke survivors were recruited from the urban setting ( 2 males (one $<45$ years and one $>45$ years) and 2 females (one $<45$ years and one $>45$ years) and similarly four (4) stroke survivors with same age and gender distribution were recruited from the rural setting. All four urban participants attained primary education as the highest while of the 4 rural participants, 2 attained primary level education and 2 secondary level education. None of the stroke survivors in urban and rural settings attained tertiary level education. Of the 4 urban stroke survivors, 2 were married and 2 were not married while none of the rural stroke survivors were married.

Four care takers of stroke survivors were recruited from the urban setting ( 2 males (one $<45$ years and one $>45$ years) and 2 females (one $<45$ years and one $>45$ years) and similarly four (4) care takers of stroke survivors with the same age and gender distribution were recruited from the rural setting. Of 4 care takers from the urban setting, 1 attained primary education as the highest while 3 attained secondary level education. On the other hand, of 4 care takers from the rural setting, 3 attained primary level education and 1 attained secondary level education. None of the care takers in urban and rural settings attained tertiary level education. Of the 4 urban care takers, 3 were married and 1 was not married. This was similar among the 4 care takers from the rural setting.

Fifteen key informants were recruited into the study with 7 (4 males, 3 females) from the urban and 8 (6 males, 2 
females) from the rural setting. The average age of the urban and rural key informants was 41.7 years (SD 7.26) with a range of 30 to 54 years and 50.5 years (SD 9.87) with a range of 28 to 60 years respectively. Of 7 urban participants, 2 attained secondary level of education and 3 attained tertiary level of education. While of the 8 rural participants, 1 attained primary level education, 3 attained secondary level education and 4 attained tertiary level education.

\subsection{Data Collection and Analysis}

16 face-to-face in-depth interviews consisting of 8 stroke patients, 8 caretakers and 15 face-to-face key informant interviews consisting of health workers, local community leaders like priests, imams, pastors, VHT leaders and local council members were conducted. Experienced qualitative researchers conducted the interviews in participants' homes or at their work places to collect narrative information on barriers to secondary stroke prevention and care. The interviews conducted in English or Luganda, a local language were audio recorded. They were later transcribed verbatim. Transcripts were independently analyzed by 2 investigators for harmonization and consistency in coding using a thematic approach where deductive and inductive codes were used to develop categories/sub themes and major themes. Atlas ti was used to analyze the data and the results reported using direct quotes.

\subsection{Ethical Approval}

The study was approved by Makerere University College of Health Sciences' higher degrees Research and Ethics Committee and Uganda National Council for Science and Technology. Additionally, permission was sought from the local authorities of the communities where the study participants were recruited. All study participants signed an informed consent form.

\section{Results}

This study sought to explore the barriers to stroke prevention and care from three categories of respondents inclusive of stroke patients, care takers and key informants. Three major domains emerged as shown in Table 1 inclusive of 1) Patient barriers with sub themes: stroke knowledge limitation, financial impediments, post-traumatic stress disorder (PTSD), pill burden constraints and adamancy when feeling better. 2) Community system barriers entailing social support constraints and community stigmatization as the sub themes and 3) Health systems barriers inclusive of inadequate human resource and drugs, inaccessibility of health care centres to patients, poor health worker-patient relationship, absence of community stroke rehabilitation centres and equipment as the sub themes.

Table 1. Categories of perceived patient, community and health system level barriers to stroke prevention and care with illustrative quotes.

\begin{tabular}{|c|c|}
\hline \multicolumn{2}{|c|}{ THEME 1: PATIENT BARRIERS } \\
\hline CATEGORIES & ILLUSTRATIVE QUOTES FROM RESPONDENTS \\
\hline Knowledge constraints & $\begin{array}{l}\text { "I do not know what causes it (stroke) and therefore I don't know how to avoid. Things like typhoid can be avoided but stroke I } \\
\text { do not know what causes it." Female care taker aged < } 45 \text { years, URBAN }\end{array}$ \\
\hline Financial constraints & $\begin{array}{l}\text { "The problem involved is that it (stroke treatment) requires a lot of money. In a month you spend 60,000 shillings yet you have } \\
\text { to eat. That sickness requires people, who are rich," Male care taker aged }>45 \text { years, URBAN }\end{array}$ \\
\hline Pill burden & $\begin{array}{l}\text { "He used to swallow and at times gets tired. Even as we talk now, he does not like taking medicine." Female care taker aged } \\
<45 \text { years, URBAN }\end{array}$ \\
\hline $\begin{array}{l}\text { Adamancy when feeling } \\
\text { better }\end{array}$ & $\begin{array}{l}\text { "They just buy a few tablets and take, when they feel better they leave the medication. When you tell them to come back for } \\
\text { review after three days to take the pressure again, they do not come back for review." Key Informant, URBAN }\end{array}$ \\
\hline \multicolumn{2}{|c|}{ THEME 2: COMMUNITY SYSTEM BARRIERS } \\
\hline CATEGORIES & ILLUSTRATIVE QUOTES \\
\hline Lack of Social support & $\begin{array}{l}\text { "If you have no one to encourage you to take drugs you can miss the drugs You can end up over sleeping and miss the timing } \\
\text { of the drugs." Male care taker aged }<45 \text { years, RURAL } \\
\text { "Me I have a problem because I have to send someone to get me drugs since I don't see but sometimes the person forgets some } \\
\text { of the medication. If I was not blind, I would go myself and get the drugs." Female Stroke Patient }<45 \text { years, RURAL }\end{array}$ \\
\hline Community stigma & $\begin{array}{l}\text { "They automatically give them names and when they quarrel with someone they abuse them and call them names," Key } \\
\text { Informant, RURAL }\end{array}$ \\
\hline \multicolumn{2}{|c|}{ THEME 3: HEALTH SYSTEM BARRIERS } \\
\hline CATEGORIES & ILLUSTRATIVE QUOTES \\
\hline $\begin{array}{l}\text { Inadequate human } \\
\text { resource and drugs }\end{array}$ & $\begin{array}{l}\text { "Most of the times they are tired, they begin attending to patients from sun rise to sunset. They have a short break and go back } \\
\text { on duty, they get tired." Key Informant, URBAN } \\
\text { "You tell them that there is hardly anything you can do for them because even their drugs are not available here." Key } \\
\text { Informant, RURAL }\end{array}$ \\
\hline $\begin{array}{l}\text { Poor Health Worker- } \\
\text { Patient Relationship }\end{array}$ & $\begin{array}{l}\text { "Patients are not easy; some patients do not appreciate the help extended to them by the health workers. They tend to overload } \\
\text { the health workers with their burdens knowing that they are health workers yet even the patients should realize that they } \\
\text { should take responsibility for their lives." Key Informant, RURAL } \\
\text { "Others (health workers) do not want to hear you out. Most of them do not want to listen to your problems. I could explain to } \\
\text { them the condition of my husband and the doctor would just say that proceed to the other point or section for medicine. } \\
\text { "Female care taker aged }<45 \text { years, URBAN }\end{array}$ \\
\hline $\begin{array}{l}\text { Absence of Community } \\
\text { Stroke Rehabilitation } \\
\text { Centres and Equipment }\end{array}$ & $\begin{array}{l}\text { "But if they can get him a wheel chair but it is better on a big compound and maybe we wheel the patient around." Female } \\
\text { care taker aged }>45 \text { years, URBAN }\end{array}$ \\
\hline
\end{tabular}




\subsection{Patient Level Barriers}

\subsubsection{Inadequate Knowledge About Stroke}

Both stroke patients and their caretakers regardless of their age bracket or social setting mentioned having limited stroke knowledge which compromised their ability to either prevent it or manage it properly. Many expressed ignorance about stroke causes and its associated symptoms and ability to be prevented or cured. Patients and care takers blamed the health workers for failing to provide them with the basic information about stroke especially when they sought treatment for various ailments in their facilities and also denied having had any stroke awareness campaign in their communities. Health workers admitted that stroke patients and the community at large were ignorant about stroke prevention and management but in defense they argued that the health education and ICT materials they received in their health facilities mainly focused on health issues that were prioritized by the ministry of Health. Unfortunately, stroke was not one of them. Below are some of the quotes from the various categories of respondents:

"Let me talk about the clinic where I go, I have never been told anything about stroke, we just go and line up then the doctor asks you what sickness you have and then he prescribes and you go and get the medicine at the pharmacy and then go home. So they do not take any initiative to educate people about the cause of stroke and the impact of stroke." Male stroke patient aged $<45$ years, URBAN

"I do not know what causes it (stroke) and therefore I don't know how to avoid it. Things like typhoid can be avoided but stroke I do not know what causes it." Female care taker aged $<45$ years, URBAN

"They (Posters on Stroke Prevention) have not come up, other posters we have depending on what the ministry is emphasizing. For instance on male circumcision, we have the posters... Yeah we have many health education programs that go on. As you can see, it (stroke) is not included as a major illness." Key Informant, URBAN

"No I have never (been sensitized about stroke); I have only been sensitized about AIDS." Male stroke patient aged > 45 years, RURAL

\subsubsection{Financial Impediments}

Financial incapacity was reported to play a major role in limiting the stroke patients' ability to access drugs recommended by health care workers, to keep clinic appointments and to adopt the recommended healthy life styles. This was mentioned by stroke patients in both urban and rural settings, although it came out profoundly from the patients in the rural setting. Respondents reasoned that much as the stroke patients were willing to adhere to prescribed drugs, they faced a challenge of accessing these drugs as they were often unavailable in the government health facilities and thus could only be accessed expensively in private health facilities. Stroke patients who were often handicapped and thus not gainfully employed, found it very hard to access medication in private health facilities. Some patients therefore resorted to taking the few doses that were either provided freely or were affordable and foregoing other prescribed medications that they could not afford to buy.

"I do not have the medication. If I had it, I would take it. When I go to Kasangati they refer me to a clinic where I have to buy the drugs. Last time I was given half of the dose because I had 1500 shillings yet they wanted 3000 shillings for the full dose." Female stroke patient $<45$ years, RURAL

"Some of the drugs, they do not have them in stock. So I just take those drugs that they give me and forego those I cannot afford to buy. If I had someone to take care of me and get me some drugs, I would feel better." Female stroke patient $>45$ years, RURAL

"Others would have come early but some have no transport, they have no money and such patients can't walk, they need transport to and from the home which may not be available.” Key Informant, RURAL

\subsubsection{Post Traumatic Stress Disorder}

Non adherence to prescribed drugs and failure to adopt healthy behaviors by both the young and older stroke patients in urban and rural settings was partly attributed to the presence of post-traumatic stress disorder symptoms such as sleeping disorders, depression and loss of self-esteem. This finding among stroke patients was confirmed by health care workers who explained that some stroke patients adopted unhealthy behaviours like smoking and alcohol drinking due to stress. Below are some of the voices of various respondents:

"I got so discouraged (by getting stroke) and resorted to boozing and I could no longer fit in with my rich friends." Male stroke patient aged $<45$ years, URBAN

"My mother got fed up of everything this time round. She even told us not to give her any medicine and injections. She told us to dump her in Mulago so that she can die there." Male care taker aged $<45$ years, URBAN

"I have no sleep because I have many thoughts. They are related to stroke because I wish I could go to places I used to go to. I used to work but now I cannot work so that makes me spend the whole night awake." Female stroke patient aged $>45$ years, RURAL

"They do not take the drugs well; they miss some days without taking the drugs such that even when I take drugs, I don't get better/healed." Female care taker aged $>45$ years, RURAL

"There are a lot of things that go around for instance he/she may be smoking because of stress or drink alcohol." Key Informant, URBAN

\subsubsection{Pill Burden Constraints}

Stroke patients, caretakers and health workers mainly from the urban setting all concurred that taking many pills on a daily basis with no hope of ever stopping them was tiresome to the patients. Such a situation was worsened by experiencing side effects while taking these drugs. Some patients consequently abandoned their drugs or missed some doses which affected the effectiveness of the drugs, leading 
to stroke recurrence.

"Taking medication daily is not easy and when you do not take it, you feel bad." Female stroke patient aged $<45$ years, URBAN.

"When I took the tablets I got a bad reaction. I took them for two days and stopped taking them, and I still have them in the house." Male stroke patient aged $>45$ years, URBAN.

"You may first quarrel for them to take the medicine yet you are dealing with a mature person who may be tired of swallowing the medicine." Key Informant, URBAN.

\subsubsection{Adamancy When Feeling Better}

Adamancy when feeling better was raised by the study respondents as having contributed to resumption of unhealthy life styles and behaviors like alcohol taking, missing clinic appointments and drugs thus leading to stroke recurrence. The following are some of the representative quotes:

"Someone may have been treated and the moment he realizes that he is feeling better, he resumes taking alcohol and even miss the appointments to hospital. Yet by the time a doctor tells you to come back for review, they have a reason as to why." Male stroke patient aged $>45$ years, RURAL

"People when they get sick and improve, they resume things they were told to stop like taking alcohol." Male care taker aged $>45$ years, URBAN.

"Me I think it is laziness and once someone realizes that he/she is healed they tend to not give a damn about the condition they had and the instructions that they were given." Key Informant, RURAL.

\subsection{Community System Level Barriers}

\subsubsection{Social Support Constraints}

In this study, lack of social support was often voiced out by respondents in all categories as a major impediment to stroke prevention and management. Adherence to drug taking, proper feeding, keeping clinic appointments, feeling accepted and coping with stroke were linked to the support a stroke patient received from his/her family and the society at large. In instances where social support was absent, stroke prevention and management became a challenge to patients. In their own words respondents observed:

"You see me since morning I have not yet been given any food but I just keep quiet and ignore that because if I have no one to give me that food that comes late, then how do I survive?" Male stroke patient aged $>45$ years, URBAN.

"The care taker may fail to give the patient medicine when he/she has gone to look for food. Sometimes failure to go back for review in hospital may be due to the fact that they have to wait for their caretakers...Even the environments they stay in make them give up on themselves causing brain damage. Many end up saying that why don't I just die. Others have nowhere to stay especially when the people looking after them are not there anymore." Key Informant RURAL.

\subsubsection{Community Stigmatisation}

Caretakers and health worker respondents in the study observed that stroke patients and their family members are subjected to name calling, disrespect and abuse not only by people in their communities but also from close family members which affects their ability to cope with stroke.

"They abuse us (stroke patient's family) and he (the stroke patient) has been nick named "Malili zone". It means that his eyes are squinted; the children despise him. When I observe that, I advise them and tell them that they will never have another daddy." Female care taker aged $<45$ years, URBAN.

"They are stigmatized by relatives and people around. It is because they cannot help themselves. They believe that they are lame, then they end up losing jobs because they cannot work due to the disability." Key Informant, URBAN.

\subsection{Health System Level Barriers}

\subsubsection{Inadequate Human Resource and Drugs}

Health workers interviewed in this study reasoned that much as they would have wanted to give stroke patients enough information concerning their disease and drugs, they were constrained by their limited numbers, the big patient load and unavailability of drugs. They reasoned that:

"... because the patient load is so big, so such information (about stroke) may be missed. As I said earlier, the doctor to patient ratio is so poor because a dispenser will work for 24 hours go in the evening then return for night duty." Key Informant, URBAN.

"Sometimes they (health workers) do not explain to the patients things concerning their sickness." Key Informant, RURAL.

"We can manage them (stroke patients) but the problem is the inability to do so due to lack of drugs." Key Informant, RURAL.

\subsubsection{Inaccessibility of Health Care Centres to Patients}

Stroke patients majorly from the rural setting made an outcry over the long distance that they had to travel to reach the health facilities in their communities. Most of them were handicapped which limited their mobility and thus required the help of other people to get them to the health facilities. The long distance travelled also had financial implications in terms of transport costs incurred which most of the stroke patients could not afford as their ability to engage in gainful employment was hampered by their physical incapacitation.

"There are some patients who live in places like Mubende who cannot even go to Kampala. It is better to get them people who can visit them in Mubende where they are. They should bring us medicine when health workers come to visit us. Male stroke patient aged $>45$ years, RURAL

\subsubsection{Poor Health Care Worker-patient Relationship}

Health care workers observed that some patients that they work on are very stubborn and not ready to take the advice concerning stroke prevention and management. On the other hand, patients and caretakers, blamed some health workers who they considered to be rude and not willing to give the patients time and information that would accelerate their recovery and prevent future disease recurrence. 
"The challenge you go through is when you advise a patient and the patient doesn't take the advice. There you get a big challenge and it is very difficult to control stroke patients. There are those who are big headed, you tell them this and they do something else. Taking medicine, the patient tells you that you give me the medicine, you give me the water then I will take it. If there is no one to attend to the patient you find the medicine there intact under the pillow. At times you tell them, don't eat this, when you move out, you find them eating that very thing. They can eat and keep quiet about it." Key Informant, URBAN.

"There is one male doctor who was very rude to me. He could not wait for me to tell him my problem, he would just write. When you would enter the room and tell him "good morning" he would reply that, "what is your problem?" and you would immediately get scared and leave him to write what he wants. Sometimes he would tell me that, "you are always complaining about paralysis, don't you get healed?" what do you expect me to tell him because that is my problem and that is what I feel I should talk to him about." Female stroke patient aged $<45$ years, URBAN.

\subsubsection{Absence of Community Stroke Rehabilitation Centres and Equipment}

Stroke patients and caretakers complained that stroke patients did not have rehabilitation centres in their communities where they can meet their peers and share information about self-management and stroke prevention. Stroke patients also wished to attend sensitization campaigns and spend time with their friends in the community but they were constrained by their inability to move. Despite some patients having wheel chairs to facilitate their movement, the wheel chairs were not customized to suit the patients' affected parts. Therefore, they had to rely on family members and well-wishers to wheel them around but these were not readily available:

"They need to give us those wheel chairs that are custom made to even aid us attend sensitization campaigns that can benefit us and even to be able to interact with others so that we can be enlightened. Many homes fail due to lack of counsel from friends, it is good to interact with people." Male stroke patients aged $>45$ years, URBAN.

"What I would think of is that the health workers tend to think that all stroke patients sit in the wheel chair. From what I have seen, stroke cuts the body into two. So instead of bringing them the usual wheel chair, they should get them a strong wheel chair depending on the side which is affected. If it hit the person on the right then they should bring a wheel chair that can be wheeled using the left hand and likewise with the left side when affected. Even it becomes easy for the care taker to look after them because the stroke patients get so lonely, spend most of the time sleeping or seated but if they have their wheel chair that they could wheel around they would go and enjoy things of their interest for instance watching football.” Key Informant, URBAN.

\section{Discussion}

This study highlighted important individual, community and health system barriers to stroke prevention and care that must be addressed to enhance successful stroke prevention and care programs.

Among the individual barriers identified in the study, the issue of paucity of knowledge about stroke causes, risk factors and its ability to be prevented or cured stood out profoundly as a major barrier that rendered stroke patients and their caretakers powerless or unarmed in the fight against stroke. Stroke knowledge gaps continue to be raised by multiple studies as a hindrance to stroke prevention and recovery in and outside Uganda [15-19] which calls for urgent inclusion of stroke messages in the mass awareness campaigns for prioritized public health issues such as HIV, immunization, male circumcision and the national day of physical activity. This empowers the populations to be able to recognize stroke risk factors, signs and symptoms that would enhance stroke prevention measures and facilitate early management. As a highly debilitating disease, stroke physically and financially incapacitates sufferers and their caretakers and yet stroke care is costly in terms of transport and drug costs involved. Contrary to study findings often cited in multiple studies [16-20], a study looking at barriers to stroke medicine adherence in Britain, made no mention of finances as a constraint to stroke medicine adherence [15] which further proves that financial impediments remain more of a problem in developing countries.

Post-traumatic stress disorders (PTSD) which are mainly sparked off by traumatic or life threatening conditions such as stroke have been found to affect patients' adherence to stroke treatment [21]. In this study, symptoms of PTSD such as feeling depressed, hopeless, constant worrying and failure to sleep were often cited by the respondents as having greatly affected their adherence to medication and/or adoption of healthy life styles. Tackling this problem requires a concerted effort where health care workers, stroke patients and their caretakers endeavor to identify any unresolved PTSD symptoms that need to be dealt with, in order to achieve a holistic stroke management strategy, as several other studies have reported similar occurrence among stroke patients [1820]. Family and community support is key especially for health conditions with long lasting physical and emotional effects on the sufferers since mobility, adherence to drugs and clinical appointments greatly depend on the social support a patient gets. In this study respondents expressed their frustrations whenever they had no one to either buy them drugs or take them to hospitals or to wheel them around Such frustrations have been mentioned in other studies where reports of stroke patients abandoning drugs due to isolation were given $[15,18,20]$. Stigmatisation of stroke patients and their loved ones through name calling has been reported in this study and multiple studies elsewhere. This lowers their self-esteem making their recovery process extremely hard. Stroke patients and caretakers felt that not having stroke 
rehabilitation centres and equipment such as customized wheel chairs greatly hindered their recovery. Formation of stroke clubs has been suggested in some studies as a way of bringing survivors together for self-help projects [18]. Health worker-patient relationship is an important component for the successful management of health problems like stroke. In this study, all categories of respondents had issues to grind with each other as often the patients blamed the health workers for handling them rudely while on the other hand the health care workers felt frustrated by patients for being non-compliant to their health instructions. Health workers partly attributed this to being overworked as a result of limited health work force which gave them little time and patience to listen to all patients' problems. Poor patient health worker relationship has often been aired out by respondents in several studies as an impeding factor to treatment seeking which calls for behavioral interventions for health workers and stroke patients.

Our study has limitations. It covered a small proportion of the population in urban and rural Wakiso district and therefore, the results may not be transferrable to other areas with varying social demographic compositions even though it employed rigorous data collection methods.

\section{Conclusion}

Stroke prevention and care programs can be successfully implemented if a holistic approach is adopted where barriers at 1) the level of stroke survivors and care takers (inadequate knowledge, financial impediments, post stroke stress disorders, pill burden and adamancy when feeling better); 2) community level (limited social support and stigmatization); and 3) health system level (scarcity of human resource and drugs, poor patient-health worker relationship, poor access to health care, lack of stroke rehabilitation centres and equipment) are addressed.

We recommend that stroke awareness messages are urgently incorporated in the mass awareness campaigns that are currently prioritized such as HIV, immunization, male circumcision and the national day of physical activity. Guidelines to tackle the individual, community and health system level barriers should be developed with involvement of key stakeholders such as the Uganda NonCommunicable Diseases and Injuries Commission to ensure better support and resource allocation at implementation of the strategies.

\section{Funding}

Research reported in this publication was supported by the Fogarty International Center, the National Heart Lung and Blood Institute, and the Common Fund of the National Institutes of Health under Award Number 5R24 TW008861. Its contents are solely the responsibility of the authors and do not necessarily represent the official views of the supporting offices.

\section{Acknowledgements}

The authors would like to thank all study participants for their valuable time and information.

\section{References}

[1] Feigin VL, Lawes CM, Bennett DA, et al. Worldwide stroke incidence and early case fatality reported in 56 populationbased studies: a systematic review. Lancet Neurol 2009; 8 (4): 355-369.

[2] Feigin VL, Forouzanfar MH, Krishnamurthi R, et al. Global and regional burden of stroke during 1990-2010: findings from the Global Burden of Disease Study 2010. Lancet 2014; 383: 245-54.

[3] Dalal PM, Malik S, Bhattacharjee M, et al. Stroke survey in Mumbai, India: incidence and 28-day case fatality. Neuroepidemiology 2008; 31 (4): 254-61.

[4] Dalal PM and Bhattacharjee M. Stroke epidemic in India: hypertension-stroke control programme is urgently needed. $\mathrm{j}$ Assoc Physicians India 2007; 55: 689-91.

[5] Katan M, Luft A. Global Burden of Stroke. Semin Neurol 2018; 38 (02): 208-211.

[6] Lozano R, Naghavi M, Foreman K, et al. Global and regional mortality from 235 causes of death for 20 age groups in 1990 and 2010: a systematic analysis for the Global burden of Diseasee Study 2010. Lancet 2012; 380: 2095-2128.

[7] WHO (2009). The Atlas of Heart Disease and Stroke. [online] Available http://www.who.int/cardiovascular_disease/resources/atlas/en/ index.html. [Accessed 1 April 2021].

[8] Mathers CD, Lopez AD, Murray CJL. The Burden of Disease and Mortality by Condition: Data, Methods, and Results for 2001. Global burden of disease and risk factors 45, 88 In Lopez AD, Mathers CD, Ezzati M, Jamison DT, Murray CJL (Eds.). Global Burden of Disease and Risk Factors. Washington DC: The International Bank for Reconstruction and Development/The World Bank Group 2006.

[9] O'Donnell MJ, Xavier D, Liu L, et al. Risk factors for ischaemic and intracerebral haemorrhagic stroke in 22 countries (the INTERSTROKE study): a case-control study. Lancet 2010; 376 (9735): 112-123.

[10] Uganda Institute for Health Metrics Evaluation (IHME). Measuring what matters, population, 1990-2100. Forecasted data based on Global Burden of Disease 2017 results. Available at http://www.healthdata.org/uganda.

[11] Connor MD, Thorogood M, Modi G, et al. The burden of stroke in Sub-Saharan Africa. Am J Prev Med 2007; 33 (2): 172-173.

[12] MRC/UVRI and LSHTM Uganda Research Unit. Stroke study to provide local data on risk factors in Uganda. Available at https://www.mrcuganda.org/news/stroke-study-provide-localdata-risk-factors-uganda.

[13] Guwatudde D, Mutungi G, Wesonga R, et al. The epidemiology of hypertension in Uganda: Findings from the National Non-Communicable Diseases Risk Factor Survey. PloS ONE 2015; 10 (9): e0138991. 
[14] Nakibuuka J, Sajatovic M, Nankabirwa J, et al. Stroke risk factors differ between rural and urban communities: population survey in central Uganda. Neuroepidemiology 2015; 44: 156-165.

[15] Jamison J, Graffy J, Mullis R, et al. Barriers to medication adherence for the secondary prevention of stroke: a qualitative interview study in primary care, Br J Gen Pract. 2016; 66 (649): e568-e576.

[16] Kaddumukasa M, Kayima J, Nakibuuka J, et al. Modifiable lifestyle risk factors for stroke among a high risk hypertensive population in Greater Kampala, Uganda; a cross-sectional study. BMC Res Notes. 2017 Dec 4; 10 (1): 675.

[17] Nakibuuka J, Sajatovic M, Katabira E, et al. Knowledge and Perception of Stroke: A Population-Based Survey in Uganda, ISRN Stroke. 2014. doi: 10.1155/2014/309106.PMID:25202472.
[18] Blixen C, Nakibuuka J, Kaddumukasa M, et al. Community Perceptions of Barriers to Stroke Recovery and Prevention in Greater Kampala, Uganda: Implications for Policy and Practice, Int J Healthc. 2017; 3 (2): 8-19.

[19] Namale G, Kawuma R. Kamacooko O, et al. Caring for a stroke patient: The burden and experiences of primary care givers in Uganda - A qualitative study. Nursing open 2019; 6 (4): 1551-1558.

[20] Pindus DM, Mullis R, Lim L, et al. Stroke survivors and informal care givers' experiences of primary care and community health care services - A systematic review and meta-ethnography Plos One 2018; 13 (2): e0192533.

[21] Kronish IM, Edmondson D, Goldfinger JZ, et al. Post traumatic stress disorder and adherence to medications in survivors of strokes and transient ischemic attacks. Stroke 2012; 43 (8): 2192-7. 